\title{
Descomposición de Materia Orgánica con Microorganismos Benéficos Magnetizados
}

\author{
Celso G. Recalde ${ }^{(1)}$, Magdy M. Echeverría ${ }^{(1)}$ y Rosa P. Castro ${ }^{(2)}$ \\ Escuela Superior Politécnica de Chimborazo, (1) Facultad de Ciencias, (2) Facultad de Recursos Naturales, \\ Panamericana Sur Km 1 1/2, Riobamba-Ecuador (e-mail: crecalde672000@yahoo.com, \\ magdymyleny@yahoo.es, rcastro@espoch.edu.ec)
}

Recibido Mar. 28, 2013; Aceptado May. 27, 2013; Versión final recibida Jul. 9, 2013

\begin{abstract}
Resumen
Se describe el comportamiento de organismos unicelulares estimulados con campos magnéticos constantes de baja intensidad generada por magnetos permanentes. Se analiza su efecto en la eficiencia de degradación en el tratamiento de residuos sólidos orgánicos, empleando un consorcio microbiano comercial, monitoreando la temperatura y a tiempos finales el $\mathrm{pH}$ y la relación carbono: nitrógeno. Del estudio se concluye que la aplicación de campos magnéticos constantes de baja intensidad a microorganismos benéficos en los procesos de descomposición de residuos sólidos orgánicos urbanos es positiva.
\end{abstract}

Palabras clave: microorganismos, campo magnético constante, descomposición biológica, residuos orgánicos

\section{Decomposition of Organic waste Material using Magnetized Microorganisms}

\begin{abstract}
The behavior of unicellular organisms stimulated with constant magnetic fields of low intensity generated by permanent magnetos is described. The effect of them on the efficiency of degradation in the treatment of organic solid wastes is analyzed, using a commercial microbial consortium, controlling the temperature and at ending times, the $\mathrm{pH}$ and the carbon-nitrogen ratio. It is concluded from the study that the application of constant magnetic fields of low intensity to beneficial microorganisms in the processes of decomposition of urban organic solid wastes is positive.
\end{abstract}

Keywords: microorganisms, static magnetic field, biological decomposition, organic wastes 


\section{INTRODUCIÓN}

La problemática de los residuos sólidos orgánicos es un tema de actualidad, que interesa a universidades y centros de investigación que buscan comprender los procesos biológicos de descomposición (Gajalakshmi y Abbasi, 2008), la influencia de parámetros como: temperatura, $\mathrm{pH}$, materia prima, microrganismos (Jahanshah y otros cinco autores, 2013), (Pérez, 2007) y (Cariello y otros tres autores, 2007). Ejecutándose experimentos que aplican tecnologías como campos electromagnéticos (Mumme, 2007), ultrasonido, microondas, agua sub y supercrítica entre otras, con resultados prometedores.

Se ha desarrollado una fuerte corriente de investigación que considera a los microorganismos benéficos como alternativa al control químico de patógenos (Cook, 1985), desde hace pocos años existen fungicidas que están siendo comercializados, creando interés por encontrar otras aplicaciones a su antagonismo con ciertos microorganismos (Zhama, Kumar y Shekhar, 2009) y (Pérez, 2007). El paso de nutrientes a nivel celular es influenciado por la distribución de los iones en el medio, lo que produce diferencias de potencial entre el interior y al exterior de la membrana (Filipič, y otros cuatro autores, 2012), la interacción de los campos electromagnéticos de baja energía con los sistemas biológicos debe ser considerada como una modificación de las propiedades eléctricas y magnéticas del sistema biológico in vivo sin cambios perceptibles a nivel cromosómico que las diferencia de las radiaciones ionizantes (Sun, 2011), (Li y otros diez autores, 2012).

Se han incrementado las evidencias de campos magnéticos como un importante método biofísico, con potencial para ejercer múltiples efectos biológicos en las células, (Muehsam y Pilla, 2009), que bajo las condiciones adecuadas la presencia de campos magnéticos puede estimular positiva (Santos, 2012), o negativamente (Filipič, y otros cuatro autores, 2012) las funciones biológicas de los seres unicelulares (Mumme y otros tres autores, 2007), (Villa, y otros tres autores, 2009). En las últimas décadas, se ha presentado mucho interés en estudiar los posibles efectos de los campos electromagnéticos en sistemas biológicos complejos y sus aplicaciones en tratamientos, diagnósticos, monitoreo y control, (Sun, 2011), los CMC de bajas intensidades muestran diferencias significativas entre las muestras tratadas y el testigo cuando la energía inducida por los magnetos es mayor que la energía térmica del sistema en el momento del tratamiento (Zborowski, 2008). En Ecuador el campo magnético terrestre es horizontal, la componente radial del geomagnetismo terrestre del potencial del dipolo es cero, mientras que, la componente tangencial es el valor máximo. Las características del dipolo magnético axial expresadas con la ecuación del potencial de un dipolo, se representa con:

$V=\mu_{4 \pi} \frac{m \cos \theta}{r^{2}}$

Para el caso de la Tierra $m$ momento dipolar, $\mu$ o permeabilidad en el vacío, $r$ la distancia desde el centro de la Tierra, $\theta$ es la colatitud medida desde el polo norte geográfico, destacando la importancia de estudiar los efectos de los CM androgénicos en la zona Ecuatorial aspecto que ha sido poco estudiado a pesar de sus múltiples implicaciones en biología, energía, nanotecnología, etc.

El propósito del trabajo es describir el comportamiento de organismos unicelulares estimulados con campos magnéticos constantes $(\mathrm{CMC})$ de baja intensidad en el tratamiento de residuos sólidos orgánicos. Se realizó un monitoreo de la temperatura tres veces al día esto es: 7h00, $12 \mathrm{~h} 00$ y $17 \mathrm{~h} 00$ durante un mes y finalmente un análisis de R:C/N y pH. De las observaciones, mediciones de campo y laboratorio, resultó positiva la aplicación de CMC de baja intensidad $1.11,0.74,0.46$ y 0.35 Gauss a microorganismos benéficos en los procesos de descomposición de residuos sólidos orgánicos urbanos.

\section{MATERIALES Y MÉTODOS}

La ESPOCH está ubicada a $2756 \mathrm{msnm}, 17 \mathrm{M} 7618039818300$, temperatura con moderadas variaciones medias mensuales durante el año, sin embargo, las mayores variaciones se producen principalmente entre el día y la noche, estableciéndose temperaturas de hasta $29,5^{\circ} \mathrm{C}$ el día y de $6^{\circ} \mathrm{C}$ bajo cero en las horas nocturnas. En los meses de marzo, abril y mayo del 2008 en que se realizó el estudio se obtuvieron temperaturas promedio mensuales de $13.2^{\circ} \mathrm{C}, 13.4^{\circ} \mathrm{C}, 13.1^{\circ} \mathrm{C}$ respectivamente (estación meteorológica ESPOCH - INAMHI.)

Entre los meses de marzo y abril del 2008, se recolectó residuos sólidos orgánicos compuestos por restos de verduras y hortalizas del mercado mayorista de la ciudad de Riobamba, transportados a la Facultad de Recursos Naturales, campus Escuela Superior Politécnica de Chimborazo (ESPOCH), donde se adecuó 13 cajones, bajo una estructura con una cubierta opaca como medida para la protección de la lluvia y de la 
radiación solar directa. Para lograr mayor uniformidad en las muestras se mezclaron los residuos sólidos orgánicos, procediéndose a colocar $15 \mathrm{Kg}$ en cada uno de los cajones, cubriéndose con una lámina de polietileno para permitir el paso de la radiación solar difusa y evitar cambios bruscos de temperatura.

El conglomerado bacteriano empleado de microorganismos benéficos (MB) es una combinación de: Trichoderma harzianum (Zhama, Kumar y Shekhar, 2009), Trichoderma viride, Trichoderma koningii, Paecilomyces lilacinus, Metarhizium anisopliae, Verticillium lecanii, Beauveria bassiana, Saccharomyces cerevisiae (Dos Santos, y otros tres autores, 2012), Bacillius thuringiensis a $2,5 \times 10^{9} \mathrm{upc} / \mathrm{mL}$, comercializado en Ecuador por el Departamento de Fitopatología-ESPOCH para control biológico de suelos.

Tabla 1: Relación $\mathrm{C} / \mathrm{N}$ y pH a los 30 días, de muestras tratadas con MB.

\begin{tabular}{|c|c|c|}
\hline & $\mathrm{R}: \mathrm{C} / \mathrm{N}$ & $\mathrm{pH}$ \\
\hline Testigo & 17.5 & 8.8 \\
\hline $75 \mathrm{~mL}$ & 16.6 & 8.7 \\
\hline $150 \mathrm{~mL}$ & 17.3 & 8.7 \\
\hline $300 \mathrm{~mL}$ & 14.5 & 7.8 \\
\hline $600 \mathrm{~mL}$ & 15 & 8.2 \\
\hline
\end{tabular}

Se tomaron 4 volúmenes diferentes de inóculo de MB: $75 \mathrm{~mL}, 150 \mathrm{~mL}, 300 \mathrm{~mL}$ y $600 \mathrm{~mL}$, se trataron por aspersión las muestras de $15 \mathrm{~kg}$ de residuos sólidos orgánicos urbanos ubicadas en cajas de tratamiento realizando 3 repeticiones de cada volumen y manteniendo un testigo referencial. Para eliminar el exceso de agua y que los MB empiecen la degradación biológica se las dejó estabilizar durante dos días. Se monitoreó la temperatura por 30 días a las $07 \mathrm{~h} 00,12 \mathrm{~h} 00$ y $17 \mathrm{~h} 00$ y al término del mes de la inoculación, se determinó $\mathrm{R}: \mathrm{C} / \mathrm{N}$, y el $\mathrm{pH}$. Obteniéndose que todos los tratamientos con diferentes volúmenes de MB inoculados por aspersión tuvieron un mejor comportamiento respecto al testigo en concordancia a los resultados obtenidos por (Cariello y otros tres autores, 2007), (Mumme, 2007), destacando que los datos de temperatura y las mediciones de $\mathrm{pH}$ y $\mathrm{R}: \mathrm{C} / \mathrm{N}$ con la dosis de $300 \mathrm{~mL}$ muestran mejores características, incluso respecto al tratamiento con $600 \mathrm{~mL}$.

La siguiente parte consiste en buscar la influencia de los CMC en el comportamiento del conglomerado de MB estudiado, tomando en cuenta que la corriente iónica existente a través de la membrana depende del gradiente electroquímico a los lados de esta, el flujo de los iones de $\mathrm{Na}^{+}$y $\mathrm{K}^{+}$son independientes a través de la membrana cuya permeabilidad depende del voltaje. El flujo de iones crea un dipolo magnético en los canales de la membrana que puede ser estimulado el microorganismo positivamente dependiendo de la intensidad y orientación del campo magnético externo (Zborowski, 2008), en poco tiempo puede haber un importante paso de iones al interior celular lo que provocaría un aumento de tamaño en las direcciones positivas de la estimulación y posiblemente una división precoz, sin embargo, es poco comprendido el comportamiento de los momentos magnéticos a ese nivel. Otros autores orientan sus investigaciones en la influencia de los CMC en la sinapsis explicando el fenómeno por las propiedades diamagnéticas de las capas de fosfolípidos de la membrana A. Rosen, 2010 en su ponencia en Xi'an, China en el evento Progress In Electromagnetics Research Symposium sobre Studies on the Effect of Static Magnetic Fields on Biological Systems, describe las significativas reducciones de la amplitud de las componentes de la sinapsis durante la exposición a CMC y estos efectos permanecen varios minutos después que el campo fue apagado. Los CMC empleados por Rosen son aplicados a una latitud planetaria diferente, por tanto respecto a Ecuador la magnitud de las componentes radiales y tangenciales del campo magnético terrestre serán diferentes.

Definido el volumen de prueba $300 \mathrm{~mL}(\mathrm{C})$ de $\mathrm{MB}$, se procede a recolectar un nuevo grupo de muestras de residuos sólidos orgánicos urbanos del mercado mayorista, realizando de manera similar el proceso, ubicando muestras de $15 \mathrm{~kg}$ de residuos sólidos orgánicos en las 13 cajas de experimentación. Los CMC se generaron con dos magnetos paralelos adecuados en forma opuesta y horizontal, sin producir campos magnéticos alternos, no inducen incremento de temperatura a diferencia de los discos que generan campos eléctrico y de los solenoides que generan campos magnéticos. Las intensidades fueron medidas en Gauss $\left(1 \mathrm{G}=10^{-4} \mathrm{~T}\right)$ con un DC-Magnetometer de AlphaLab Inc. /USA con certificado de calibración válido hasta el año 2017. Se ajustò el equipo encerandolo para eliminar de la medida el ruido ambiental y el campo terrestre, de esta manera se cuantificó el campo producido por los magnetos, ubicando la sonda con el sensor paralelo a las placas metálicas como indica el manual. 
Se estimulan los $300 \mathrm{~mL}$ de MB dispuestos en un contenedor de vidrio, ubicando el envase con los MB en la región homogénea del campo (Zúñiga, Cuero y Peña, 2011) durante 5 minutos. Donde las intensidades del CMC decrecen según el cuadrado de las distancias. Se repitió el proceso para cada intensidad del campo, por 3 ocasiones y el testigo con $300 \mathrm{~mL}(\mathrm{C})$ de $\mathrm{MB}$ sin tratamiento (Dos Santos y otros tres autores, 2012). Se aplicaron 4 intensidades de $\mathrm{CMC}$ con tres repeticiones y el testigo. Las intensidades del campo corresponden a: $1.11,0.74,0.46$ y 0.35 Gauss. Se inoculó por aspersión los $300 \mathrm{~mL}$ de MB magnetizados con 4 intensidades de CMC con 3 repeticiones a las 12 muestras de $15 \mathrm{~kg}$ de residuos sólidos orgánicos urbanos, manteniendo el testigo con $300 \mathrm{~mL}$ de $\mathrm{MB}$ sin magnetizar. La temperatura fue monitoreada a las 07h00, $12 \mathrm{~h} 00$ y $17 \mathrm{~h} 00$ y al término del mes de la inoculación, se determinó R: C/N, y el pH.

\section{RESULTADOS Y DISCUSION}

La influencia producida por diferentes intensidades de pequeños CMC aplicados por 5 minutos, sobre la actividad biológica al interior de residuos solidos orgánicos urbanos tratadas con volúmenes diferentes de $\mathrm{MB}$, fue evaluada monitoreando los datos de temperatura en las muestras y un análisis de $\mathrm{pH}$ y $\mathrm{R}: \mathrm{C} / \mathrm{N}$, descritos en la Tabla 1. Se realizó el anova de un factor al 0.05 nivel de significancia mostrando que las diferencias son significativas entre el testigo y los tratamientos. El volumen de MB que mejor comportamiento presentan en la descomposición orgánica de las muestras de $15 \mathrm{~kg}$ de residuos sólidos orgánicos urbanos fue el de $300 \mathrm{~mL}$ de $\mathrm{MB}$, La relación $\mathrm{C} / \mathrm{N}$ en las muestras se redujeron de modo similar al comportamiento mostrado por Cariello, 2007.

Definido el volumen de $300 \mathrm{~mL}$ de MB se procede a aplicar diferentes intensidades de pequeños CMC esto es: $1.11,0.74,0.46$ y 0.35 Gauss durante 5 minutos a cada muestra de $300 \mathrm{~mL}$ de MB. Se midió las temperaturas al interior de las muestras de residuos solidos orgánicos urbanos siguiendo el proceso de descomposición tres veces al día: 7h00, $12 \mathrm{~h} 00$ y 17h00. Se aplicó anova de un factor al 0.05 nivel de significancia para la relación $\mathrm{C} / \mathrm{N}$ donde el $\mathrm{f}$ calculado 0,66 es menor que $f$ tabulado 0,22 y para el pH el $f$ calculado 2,76 es menor que f tabulado 19,24 mostrando que son estadísticamente iguales. Con la temperatura se hizo un análisis de varianza de un factor entre tratamientos y entre días que se monitoreo la temperatura indicando que los $300 \mathrm{~mL}$ de MB tratados con pequeños CMC de 0.74 Gauss presentan mejores resultados para la descomposición biológica de los desechos solidos orgánicos urbanos tratados en este trabajo acorde con lo presentado en la cachaza (Zúñiga, Cuero y Peña, 2011).

Tabla 2: Relación $\mathrm{C} / \mathrm{N}$ y pH a los 30 días de muestras tratadas con MB magnetizados.

\begin{tabular}{|c|c|c|}
\hline & $\mathrm{pH}$ & $\mathrm{R}: \mathrm{C} / \mathrm{N}$ \\
\hline Testigo 300mL & 8 & 14.0 \\
\hline $300 \mathrm{~mL}+1.11$ Gauss & 7.5 & 26.3 \\
\hline $300 \mathrm{~mL}+0.74$ Gauss & 8 & 41.3 \\
\hline $300 \mathrm{~mL}+0.46$ Gauss & 7.5 & 23 \\
\hline $300 \mathrm{~mL}+0.35$ Gauss & 8.5 & 32.7 \\
\hline
\end{tabular}

El estimulo inicial provocado en los 5 minutos de permanencia de los $300 \mathrm{~mL}$ de $\mathrm{MB}$ al interior del $\mathrm{CMC}$ con las cuatro intensidades aplicadas mejora la actividad biológica, acelerando la descomposición de los residuos solidos orgánicos urbanos resultado similar a Small, Hüner y Wan, 2012, este estimulo se presentó hasta el día tres, el comportamiento de los tratamientos y el testigo se uniformizó en adelante. La actividad biológica mantenía una temperatura mayor a la ambiental mientras existían nutrientes, esto es hasta aproximadamente el día 21, ver Figura 1,

La curva de crecimiento bacteriano influye en el comportamiento de la temperatura, considerando que la fase de recuperación y adaptación al nuevo medio ocurre entre los dos días transcurridos desde la inoculación por aspersión y la primera medición de la temperatura; el día uno correspondería a la etapa exponencial 0 fase estacionaria por cuanto se presentaron los valores de temperatura mayores deduciéndose que los microrganismo han crecido y se han dividido en el medio. Las diferencias de temperatura entre los medios de prueba y la temperatura ambiente indica que la actividad biológica en los primeros tres días es mayor, disminuyendo al desaparecer la inercia del estímulo inicial y bajar la disponibilidad de nutrientes. Las mediciones de la temperatura al final del seguimiento se equiparan a la temperatura ambiental correspondiendo al declive o muerte última etapa del crecimiento dado el detrimento ambiental, escases de nutrientes, aparecimiento y acumulación de desechos tóxicos. 


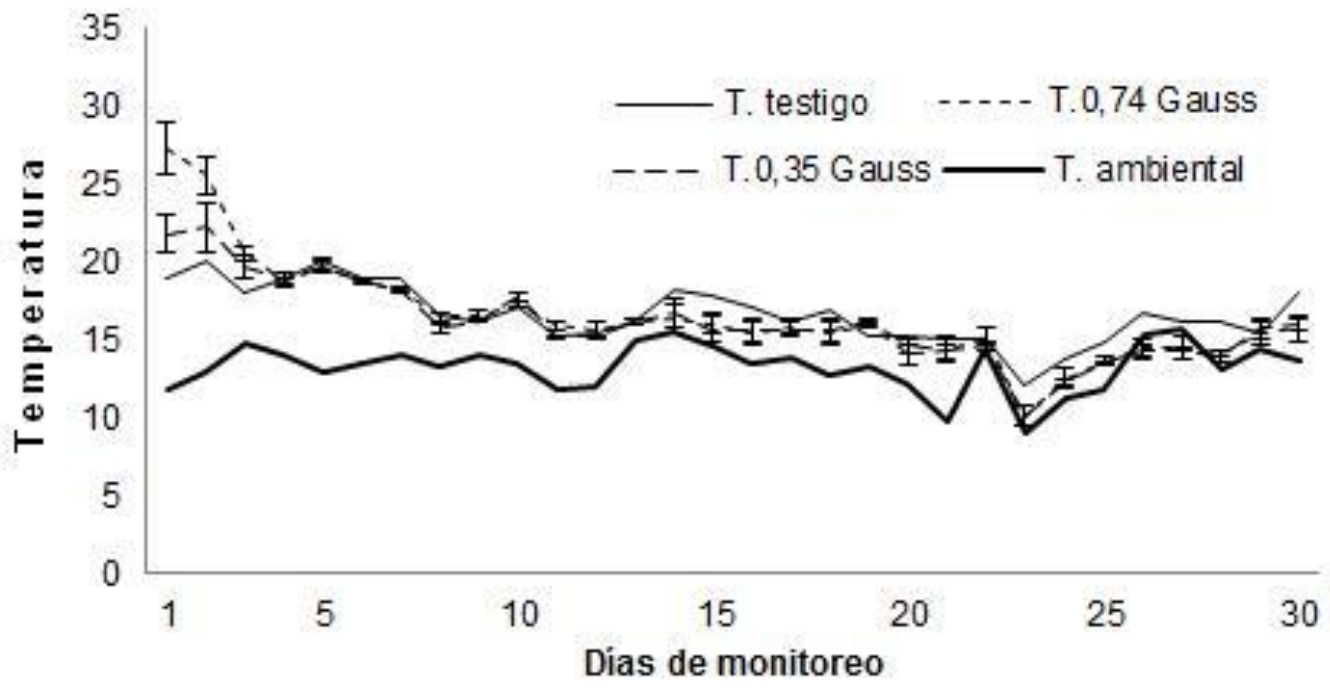

Fig. 1: Comportamiento de la temperatura en grados Celsius en los 30 días de monitoreo a las 07h00.

Tabla 3: Temperatura ambiental, del testigo y el promedio de las muestras tratadas con CMC en grados Celsius (T testigo, MA 1,11 Gauss, MB 0,74 Gauss, 0,46 Gauss, 0,35 Gauss).

\begin{tabular}{|c|c|c|c|c|c|c|c|c|c|c|c|c|c|c|c|}
\hline \multirow[b]{2}{*}{ D } & \multicolumn{5}{|c|}{ 07h00 } & \multicolumn{5}{|c|}{$12 \mathrm{~h} 00$} & \multicolumn{5}{|c|}{$17 \mathrm{~h} 0$} \\
\hline & $T$ & IA & MB & 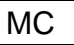 & & $T$ & AA & MB & $\mathrm{MC}$ & IVID & 1 & MA & $M B$ & 1C & 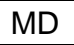 \\
\hline 1 & 9,0 & 9,8 & 27,3 & & & & & & & & & & & & \\
\hline 2 & & 18 & & & & & & & & & & & & & \\
\hline 3 & & 0 & & & & & & & & & & & & & \\
\hline 4 & & 3,9 & & & & & 9,9 & & & & & & & & \\
\hline 5 & & 9,8 & & & & & 9,9 & & & & & & & & \\
\hline 6 & ,0 & 18, & 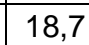 & & 8 & & 0 & & & 18,3 & $\overline{9,5}$ & 8 & & & 3,8 \\
\hline 7 & & & 18 & & 18,2 & & & & & & & & & & 8,2 \\
\hline 8 & & & 16 & & 15,7 & & 72 & & & & 8,3 & & & & 8,0 \\
\hline 9 & & & 16 & & 16,2 & & 8 & & & & & 8 & & & 8,9 \\
\hline 10 & 0 & 17 & 17 & 17 & 17,8 & & 17 & & & 17 & & 1 & & & 7,2 \\
\hline 11 & 83 & 15 , & 15 & - & 15,1 & & 16,6 & & & & & 1 & & & 3,1 \\
\hline 12 & 81 & -5 & 15 & & 15,2 & & 78 & & & & & & & & 7,8 \\
\hline 13 & & & 16 & & 16,2 & & 18,4 & & & & & & & & 8,7 \\
\hline 1 & & & & & 16,6 & & & & & & & & & & 3,2 \\
\hline 1 & & & & & & & & & & & & & & & \\
\hline 1 & & & & & & & & & & & & & & & \\
\hline 1 & & & & & & & & & & & & & & & 3 \\
\hline & & & & & & & & & & & & & & & \\
\hline & & & & & & & & & & & & & & & \\
\hline 2 & & & & & 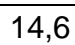 & & & & & & & & & & \\
\hline 2 & & & & & 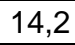 & & & & & & & & & & \\
\hline 22 & & & & & 14 & & & & & & & & & & \\
\hline 23 & & & & & - & & & & & & & & & & \\
\hline 24 & & & & & 12 & & & & & & & & & & 6 \\
\hline 25 & 1 & & & & 13 & & & & & & & & & & 14,9 \\
\hline 26 & 16,7 & 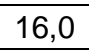 & & & 14,2 & & 1 & & & & & & & & 2,8 \\
\hline 27 & & 16 & & & 14 & & 20 & & & & & & & & 3,8 \\
\hline 28 & & 15 & & & 14 & & 18 & & & & & & & & 2,9 \\
\hline 29 & & 10 & & & & & & & & & & & & & 3,5 \\
\hline 30 & 7,9 & , 6 &, 7 & , 0 & 5,9 &, 4 & 0,6 & , & 18,4 & 18,8 & 3,2 & 13 & 12,6 & 12,5 & 2 \\
\hline
\end{tabular}


Los microorganismos son en una mezcla de $\mathrm{n}$ volúmenes de sustancias con diferente susceptibilidad magnética por unidad de volumen, en este trabajo se emplea un consorcio microbiano de MB donde los efectos de los CMC serán diferentes para cada microorganismo, incluso no será similar la incidencia al interior de estos seres unicelulares. Sin embargo, se puede aproximar que la susceptibilidad magnética de todo el volumen como el promedio ponderado de las susceptibilidades individuales, medir los parámetros macro e inferir el fenómeno a nivel micro.

\section{CONCLUSIONES}

Los efectos en Ecuador del estimulo de pequeños campos magnéticos constantes aplicados por cinco minutos a $300 \mathrm{~mL}$ de MB es reversible, el proceso de descomposición de las muestras de $15 \mathrm{~kg}$ de residuos sólidos orgánicos urbanos es mayor que el testigo hasta el tercer día como indica el comportamiento de la temperatura de los tratamientos, testigo y temperatura ambiental Figura 1.

Los microorganismos tratados con CMC van perdiendo la inercia magnética del estímulo al disminuir la energía asociada al momento magnético inducido, al cuarto día deja de ser perceptible como muestra el comportamiento de las temperaturas de las muestras tratadas y del testigo con valores similares Figura1.

El mayor consumo inicial de nutrientes por los MB tratados con $\mathrm{CMC}$ en los primeros tres días del monitoreo resulta una mayor perdida de temperatura respecto al testigo que conservó mayor reserva de nutrientes en los últimos días del monitoreo Figura1.

No se mostraron diferencias significativas en el $\mathrm{pH}$ y en la R:C/N entre las muestras tratadas y el testigo a los treinta días del monitoreo indicando que los primeros días de evidencias (temperatura) de influencia magnética fueron anuladas, por las interacciones ambientales.

\section{AGRADECIMIENTOS}

Al Dr. Rodolfo Pasinetti representante de la fundación italiana CDF, Ing. Rubén Ruiz funcionario del ex CONESUP por el apoyo prestado al grupo de Energías Alternativas y Ambiente de la ESPOCH.

\section{REFERENCIAS}

Cariello, M. y otros tres autores, Inoculante de microorganismos endógenos para acelerar el proceso compostaje de residuos sólidos urbanos, J. Soil Sc. Plant Nutr, ISSN 0718-2791 (en línea), 7(3), 26-37 (2007). http://www.scielo.cl/scielo.php. Acceso: 22 de Febrero 2013.

Cook, R. J., Biological control of plant pathogens: theory to application. Phytopathology: 75(1), 25-29 (1985).

Dos Santos, L. y otros tres autores, Glutathione production using magnetic fields generated by magnets, Braz. arch. biol. Technol, ISSN 1516-8913 (en línea), 55(6), 921-926 (2012). http://www.scielo.br/scielo.php. Acceso: 23 de Febrero 2013.

Filipič, J. y otros cuatro autores, Effects of low-density static magnetic fields on the growth and activities of wastewater bacteria Escherichia coli and Pseudomonas putida. Bioresource Technology, ISSN 0960-8524 (en línea), 120(1), 225-232 (2012). http://www.sciencedirect.com/science. Acceso: 25 de Febrero 2013.

Gajalakshmi, S. y Abbsi S., Solid waste management by composting: State of the art, Critical Reviews in Environmental Science and Technology: 38(5), 311-400 (2008).

Jahanshah, G., y otros cinco autores, Enhancing compost quality by using whey-grown biosurfactantproducing bacteria as inocula, Annals of Microbiology: 63(1), 91-100 (2013).

Muehsam, D., y A. Pilla. A Lorentz model for weak magnetic field bioeffects: part l-thermal noise is an essential component of $A C / D C$ effects on bound ion trajectory. Bioelectromagnetics: 30(6), 462-475, (2009).

Mumme, J., y otros tres autores, Magnets hold microorganisms in the fermentor. Higher efficiency of biogas plants with magnetic particles. Neue Landwirtschaft, ISSN 0863-2847 (en línea), 11(1), $94-96$ (2007). http://www.dlv.de. Acceso 20 Enero 2013. 
$\mathrm{Li}, \mathrm{Y}$. y otros diez autores, Low Strength Static Magnetic Field Inhibits the Proliferation, Migration and Adhesion of Human Vascular Smooth Muscle Cells in a Restenosis Model Through Mediating Integrins $\beta 1$ FAK, Ca2+ Signaling Pathway. Annals of Biomedical Engineering: 40(12), 2611-2618 (2012).

Pérez, S. y otros tres autores, Uso de Enzimas de Tipo Ureasa en el Tratamiento de Aguas Residuales con Alto Contenido en Nitrógeno Orgánico. Información Tecnológica, ISSN 0718-0764 (en línea), 18(5), 41-48 (2007). http://www.scielo.cl/scielo.php. Acceso 2 Julio 2013.

Small, D., Hüner, N. y Wan, W., Effect of static magnetic fields on the growth, photosynthesis and ultrastructure of Chlorella kessleri microalgae, J. Bioelectromagnetics, ISSN 1521-186X (en linea), 33(4), 298-308 (2012). http://dx.doi.org/10.1002/bem.20706. Acceso 22 de Febrero 2013.

Sun, P., y otros ocho autores, Increase in cortical pyramidal cell excitability accompanies depression-like behavior in mice: a transcranial magnetic stimulation study. J. Neurosci: 31(45), 16464-16472 (2011).

Villa, W. y otros tres autores, Calculo del Campo Magnético en el Interior de Edificaciones con Sistemas de Protección Contra Rayos. Información Tecnológica, ISSN 0718-0764 (en línea), 20(3), 45-54 (2009). http://www.scielo.cl/scielo.php. Acceso 2 Julio 2013.

Zborowski, M. y Chalmers J., Magnetic Cell Separation, Laboratory Techniques in Biochemistry and Molecular Biology, Elsevier, ISBN: 978-0-444-52754-7, 32(1), 473, Amsterdam, (2008).

Zhama, K., A. Kumar y R. Shekhar, Morphological, Biochemical and Molecular Characterization of Trichoderma harzianum Isolates for their Efficacy as Biocontrol Agents, J. Phytopathology: 157(1), 51-56 (2009).

Zúñiga, O., R. Cuero y J. Peña, Estimulação com variável do campo eletromagnético benéficos utilizados microrganismos para melhorar o cachaça use como biofertilizantes. Rev.Bio.Agro, ISSN 1692-3561 (en línea), 9(2), 150-158 (2011). http://www.scielo.org.co/scielo.php. Acceso: 23 de Febrero 2013. 
